device does not perform as it should; the sooner they report it to the FDA, the faster the agency can analyze the problem and take corrective action," said FDA Commissioner Dr. David A. Kessler.

An important part of MEDWatch is a simplified reporting form-a self-addressed, one-page form that can be folded and mailed postage-free. In addition, the agency has established a special 24-hour toll-free telephone line, (800) FDA-1088, for adverse event information. Health professionals also will be able to file reports on a computer.

\section{Joint Commission to Make Hospital Performance Information Available to Public}

Starting next year, the Joint Commission on Accreditation of Healthcare Organizations for the first time will release information to the public on how hospitals meet specific performance standards. Commission President Dennis S. O'Leary said, "This is a landmark issue for us ... it is very much in line with the reform environment. The change in the Joint Commission's confidentiality and disclosure policy, approved recently by the commissions board, recognizes the accrediting agency's obligation to share information with patients, purchasers, and other stakeholders in healthcare delivery systems."

Under the new policy, standards-compliance ratings in such areas as nursing care, infection control, patient rights, and life safety will be provided on a comparative basis. Also available for comparison will be facilities' overall summary grid scores, which are the basis for accreditation decisions.

Healthcare organizations will be able to review and comment on the data in the Joint Commission performance reports prior to their publication, but official survey reports will remain confidential.

FROM: Trustee magazine. American Hospital Association, July 1993.

\section{CDC Clarifies Recommendation for Use of Bleach to Disinfect Needles}

The CDC clarified its April 19, 1993, joint bulletin regarding the use of bleach for disinfecting needles and syringes, including the recommendation for the use of full-strength household bleach. In a letter signed by Dr. James Curran, Associate Director for HIV/AIDS, the CDC explained that it recommended full-strength bleach to disinfect needles and syringes because of the difficulty of cleaning the interior of needles and syringes and the use of needles and syringes for parenteral injection.

This recommendation does not change the recommendations for situations encountered in healthcare settings, such as contamination of environmental surfaces with blood. The CDC recommendation for disinfecting environmental surfaces continues to include the use of a 1:100 dilution of household bleach (or $1 / 4$ cup bleach to 1 gallon tap water) (See $M M W R$ 1993;46:1-18S; August 21, 1987.)

\section{Lifetime AIDS Treatment Cost: $\$ 119,274$}

The lifetime cost of treating a person with HIV disease, assuming treatment begins immediately after infection and diagnosis, averages \$119,274, Dr. Fred Hellinger of the Agency for Health Care Policy and Research recently reported.

The figure was calculated by multiplying the average monthly cost of care in each of four stages of HIV disease by the average number of months the infected person spends in each stage. Dr. Hellinger's study was based on self-reported interviews and data every three months from 1,164 respondents from March 1991 through August 1992 at 26 sites in 10 cities in the United States. Costs included inpatient care, outpatient visits, home healthcare, long-term care, and drugs, both self-paid and covered by insurance. The survey assumed an average of 10.3 years from infection until progression to AIDS and a 25 -month survival time from then until death.

Hellinger said the study was not random and thus the lifetime costs represented the "upper bounds" for the cost of treatment. The study found that the cost of treating a person with AIDS, which has risen rapidly in the past, has fallen as a result reducing the use of inpatient hospital services.

FROM: Hellinger FJ. The lifetime cost of treating a person with HIV infection. JAMA 1993;270:474-478.

\section{Government Considers Relaxing CLIA Regulations}

The federal government now is looking at proposals for relaxing physician office laboratory regulations under the Clinical Laboratory Improvement Acts (CLIA). Although the Clinton administration vowed to ease some of the more burdensome requirements, it appears that the proposals for easing the rules are driven by the lack of money rather than a desire to limit the burden on physicians. The current plan is to let "low-volume" and "low-risk" labs perform selfassessments rather than submit to on-site surveys by government inspectors.

CLIA set up four categories of labs based on test complexity. It is unclear how the government would define low-risk labs for the purpose of this new proposal. However, it is assumed that the tests they perform would fall into CLIA's "moderately complex" category.

Labs doing high-complexity testing still would be 
subject to regular on-site inspections. Those that fall into the waived or physician microscopy categories are already exempt. Low-volume labs are generally thought to be those that perform fewer than 2,000 tests annually.

Under the current proposal, backed by top Health and Human Service (HHS) officials, labs that pass an inspection could waive their next one. However, no labs would be able to waive two inspections in a row.

The law, which took effect last September, requires all labs to be surveyed every two years to make sure they are in compliance with CLIA regulations. By law, CLIA was to have been entirely user fee-funded through charges for registration, certification, and compliance. The Health Care Financing Administration (HCFA) was to use this money to pay all CLIA's overhead. But federal officials have said that CLIA fees proved insufficient to meet the program's operating expenses, requiring HCFA to underwrite the development and implementation costs.

Federal officials are meeting with key members of Congress to discuss the proposed CLIA changes. HCFA hopes to make these changes administratively to avoid a legislative battle with members of Congress who oppose an easing of the rules.

FROM: A merican M edical News. August 9, 1993.

\section{Cost Savings and Health Benefits Prompts Medicare Coverage of Influenza Vaccine}

A four-year congressionally mandated demonstration project has demonstrated health and economic benefits of influenza vaccination and has prompted coverage for influenza vaccine for all Medicare beneficiaries. In the last year alone of the Medicare Influenza Vaccine Demonstration, overall influenza vaccination levels almost exceeded the national health objective for the year 2000 of $60 \%$ vaccine coverage among noninstitutionalized persons $\geqslant 65$ years.

Case-control studies of vaccine effectiveness in preventing hospitalization for pneumonia were conducted during the demonstration. These studies estimated that influenza vaccine was $31 \%$ to $45 \%$ effective in preventing hospitalization for any pneumonia during the 1989-90, 1990-91, and 1991-92 influenza season.

The results of the cost-effectiveness analysis varied because of the variability of influenza from season to season in causing disease outcomes and the difficulty of attributing these outcomes to influenza. Nonetheless, provision of influenza vaccine was costeffective for Medicare and may be cost-saving, depending on the effectiveness of the vaccine in reducing pneumonia hospitalizations and deaths and the level of vaccine coverage.

The demonstration project's success in vaccine delivery resulted from focused interventions to overcome common barriers to adult vaccination, including the absence of a comprehensive vaccine delivery system, limited reimbursement mechanisms, and lack of vaccination programs where adults congregate.

FROM: Final results: Medicare influenza vaccine demonstration-selected states, 19881992. M M WR 1993;42:601-604.

\section{AIDS Rates Increases Are Greater Among Women}

Reported AIDS cases among women in 1992 continued to increase at a faster rate than among men, and for the first time, more than half the cases among women were the result of heterosexual contact. From 1991 to 1992 , the increase in reported cases among women was $9.8 \%$, compared to $2.4 \%$ for men. Although the proportionate increase in cases attributed to heterosexual contact was greater for men than for women in 1992 (26.3\% versus $11.5 \%)$, women accounted for a majority of the persons infected through heterosexual contact (59.4\%). For the second consecutive year, the number of reported cases among homosexual/bisexual men decreased during 1992.

The CDC stated that "the steady increase in heterosexually acquired AIDS cases among men and women underscores the need to improve understanding of the factors that influence the adoption of safer sexual practices ... and how these factors vary in different population subgroups." The CDC also commented that because injecting drug users and men who have sex with men continue to account for $80.5 \%$ of the cases, prevention efforts targeting these populations must remain a priority and interventions targeting persons at increased risk for heterosexual transmission must be strengthened.

FROM: Update: AIDS, United States, 1992. M M W R 1993;42:547-551,557. 